\title{
Thermal biomimetic sensing as a tool for point-of-care bacteria detection
}

\author{
$\underline{\text { K. Eersels }}{ }^{1}$, B. van Grinsven ${ }^{1}$, R. Rogosic ${ }^{1}$, J. Lowdon ${ }^{1}$, B. Heidt ${ }^{1}$, H. Diliën ${ }^{1}$, E. Steen Redeker ${ }^{1}$, \\ M. Peeters ${ }^{2}$, P. Cornelis ${ }^{3}$, P. Wagner ${ }^{3}$, T.J. Cleij ${ }^{1}$ \\ ${ }^{1}$ Maastricht University, Maastricht Science Programme, PO Box 616, 6200 MD, Maastricht, the Netherlands, \\ ${ }^{2}$ Manchester Metropolitan University, Faculty of Science \& Engineering, Div. of Chemistry \& Environmental Science, John \\ Dalton Building, Chester Street, M15GD, Manchester, United Kingdom. \\ ${ }^{3}$ KULeuven, Department of Physics and Astronomy, Soft-Matter Physics and Biophysics Section, Celestijnenlaan 200D, B- \\ 3001, Leuven, Belgium \\ kasper.eersels@maastrichtuniversity.nl
}

\begin{abstract}
This work introduces a novel bacterial identification assay based on thermal wave analysis through surface-imprinted polymers (SIPs). A novel thermal sensing platform; coined thermal transport analysis (TWTA) that has been previously used for the detection of dopamine in banana juice was combined for the first time with SIPs. The technique, based on the analysis of a propagation of a thermal wave through functionalized surfaces, has previously been shown to be more sensitive than the previously developed heat-transfer method. SIP-coated aluminum chips were imprinted with Escherichia coli and used for the detection of bacteria in spiked urine samples. The proposed sensor platform was able to detect bacteria in concentration regimes relevant for urinary tract diagnosis (UTI), illustrating the diagnostic potential of the thermal detection platform and its combination with synthetic receptors.
\end{abstract}

Key words: surface-imprinted polymers, thermal wave transport analysis (TWTA), bacterial detection, urinary tract infection, diagnostic tool

\section{Introduction}

Over the past decade, various biomimetic sensor platforms based on synthetic, bioinspired receptors have been developed. In terms of macromolecular targets, surfaceimprinted polymers (SIPs) have emerged as interesting candidates for incorporation into point-of-care detection systems. Until now, SIPbased sensors have been mainly combined with quartz crystal microbalances readout platforms. $^{1}$

In 2012, a surprisingly versatile tool for labelfree sensing was developed; the so-called heattransfer method (HTM). In previous work, SIPbased detection of bacteria was demonstrated using this thermal readout strategy. ${ }^{2,3}$ Although the results of these experiments were promising, the relatively narrow dynamic range and limited sensitivity of the sensor limited its application in e.g. screening healthcare surfaces for the presence of bacteria in hygiene management.

Therefore, the sensitivity of the thermal sensing principle has been improved in this study by analyzing the propagation of a thermal wave through the receptor layer rather than a constant current (thermal wave transport analysis or TWTA). The improved methodology allows for selective bacteria detection in urine in concentrations relevant for diagnosis of urinary tract infection (UTI). ${ }^{4}$

\section{Methods}

Aluminum chips were coated with polyurethane and imprinted with various target-cells to create SIPs for E. coli. A thermal current with an average temperature of $37^{\circ} \mathrm{C}$, an amplitude of $0.1^{\circ} \mathrm{C}$ and a frequency of $0.03 \mathrm{~Hz}$ was send through the SIP-coated chip and the phase shift of the transmitted wave was analyzed by TWTA in function of the concentration of bacteria in the sample under analysis.

\section{Results}

The SIP layer was exposed to urine samples spiked with increasing concentrations of $E$. coli. The results indicate that the observed phase shift on the transmitted concentration increases in function of the concentration of bacteria (Figure 1). 


\section{Discussion}

Binding of bacteria to the SIP layer increases the thermal resistance of the solid-liquid interface as they displace the urine that was previously inside the binding cavities (waterbased, good thermal conductor). Therefore, the heat wave will propagate less smoothly through the SIP-coated aluminum chip, leading to the observed phase shift in Figure 1. The sensor is able to detect bacteria in concentrations as low as $10,000 \mathrm{CFU} \mathrm{mL}-1$, which is in line with concentrations observed in urinary tract infection. Therefore, these results illustrate the potential application of the current sensor platform in UTI diagnosis.
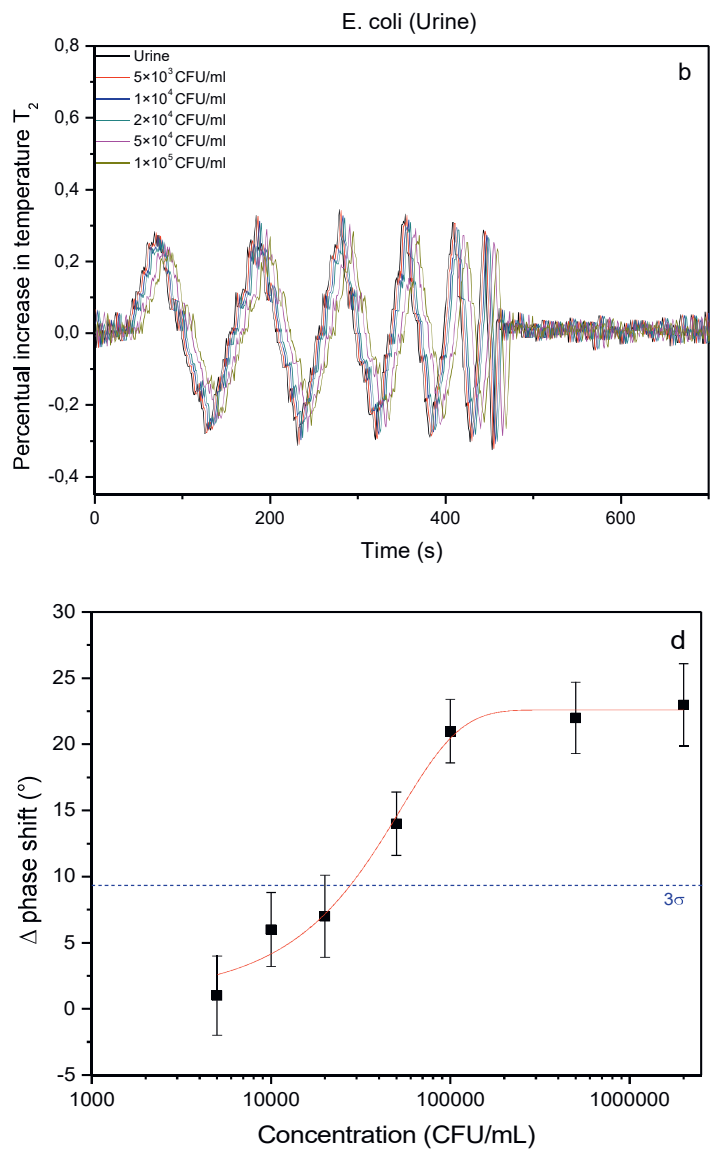

Fig. 1. Serotonin detection in whole blood samples using the thermal detection platform. Upon addition of blood, the temperature inside the flow cell rises as a result of blood being a better conductor than air. The increase observed in the MIP channel is lower as the binding of serotonin to the MIP increases the heat-transfer resistance of the solid-liquid interface

\section{References}

[1] K. Eersels, et al. A Review on Synthetic Receptors for Bioparticle Detection Created by Surface-Imprinting Techniques-From Principles to Applications. ACS Sens. 1, 1171-1187 (2016); DOI: 10.1021/acssensors.6b00572

[2] B. van Grinsven, K. Eersels, et al. Label-Free Detection of Small Organic Molecules by
Molecularly Imprinted Polymer Functionalized Thermocouples: Toward In Vivo Applications. ACS Sens., 2, 583-589 (2017); DOI: 10.1021/acssensors.7b00104

[3] B. van Grinsven, K. Eersels et al. SIP-Based Thermal Detection Platform for the Direct Detection of Bacteria Obtained from a Contaminated Surface. Phys. Stat. Solidi (2018); DOI: 10.1002/pssa.201700777

[4] E. Steen Redeker, K. Eersels, et al. Biomimetic Bacterial Identification Platform Based on Thermal Wave Transport Analysis (TWTA) through Surface-Imprinted Polymers. ACS Infect. Dis. 3, 388-397 (2017); DOI: 10.1021/acsinfecdis.7b00037. 